\title{
Ion escape from the upper ionosphere of Titan triggered by the solar wind
}

W. M. Moslem ${ }^{1,2,3, a}$, S. Salem ${ }^{1,4, b}$, R. Sabry ${ }^{5, c}$,

M. Lazar ${ }^{3,6, d}$, R. E. Tolba ${ }^{2, e}$, and S. K. El-Labany ${ }^{7, f}$

${ }^{1}$ Department of Physics, Faculty of Science,

Port Said University, Port Said 42521, Egypt

${ }^{2}$ Center for Theoretical Physics, British University in Egypt (BUE), El-Shorouk City, Cairo, Egypt

${ }^{3}$ Institut für Theoretische Physik, Lehrstuhl IV: Weltraum- und Astrophysik,

Ruhr-Universität Bochum, D-44780 Bochum, Germany

${ }^{4}$ Basic and Applied Science Department,

College of Engineering and Technology,

Arab Academy for Science and Technology (AAST), Port Said, Egypt

${ }^{5}$ Department of Physics, College of Science and Humanitarian Studies,

Sattam Bin Abdul Aziz University, Alkharj, Saudi Arabia

${ }^{6}$ Center for Plasma Astrophysics, KU Leuven,

Celestijnenlaan 200B, B-3001 Leuven, Belgium and

${ }^{7}$ Department of Physics, Faculty of Science,

Damietta University, New Damietta 42521, Egypt

(Dated: June 22, 2019) 


\begin{abstract}
The observed escape of ions from the ionosphere of Titan suggests a potential role of the solar wind in the interaction with the upper atmospheric layers. We investigate such a plausible scenario using a hydrodynamic fluid approach for the plasma expansion in the upper ionosphere of Titan, combining (Maxwellian) electrons and three different species of positive ions which interact with the solar wind electrons and protons. Using a self-similar transformation, numerical analysis is performed to solve the basic equations, and characterize the plasma density, velocity, and electric potential during the expansion. It is found that increasing the solar wind proton number density leads to a reduction the ion escape, while the effect of electrons is opposite stimulating the ion escape. Moreover, the expansion domain does not change for more energetic protons. The heavier ions has the leading role in controlling the ion escape, i.e. by comparison to $\mathrm{CH}_{5}^{+}$ions, $\mathrm{C}_{2} \mathrm{H}_{5}^{+}$ions appear to have a more influence in the loss from Titan ionosphere. For a higher temperature contrast between ions and electrons, the depletion rate of the density increases and the ions move faster, leading to a higher ionic loss.
\end{abstract}

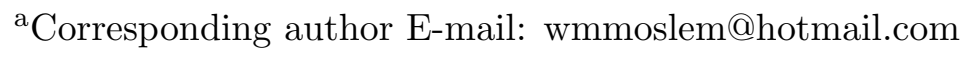

${ }^{b}$ E-mail: samy_egy007@yahoo.com

${ }^{\mathrm{c}}$ E-mail: sabryphys@yahoo.com

${ }^{\mathrm{d}}$ E-mail: mlazar@tp4.rub.de

eE-mail: reda.tolba@bue.edu.eg

${ }^{\mathrm{f}}$ E-mail: skellabany@hotmail.com
}

PACS numbers: 


\section{INTRODUCTION}

Solar wind results from a continuous outflow of mass and energy from the Sun's surface, and is enhanced during the energetic events like coronal mass ejections. Roughly one million tonnes of hydrogen are released per second in space, with energy in the range $1.5-10$ $\mathrm{keV} /$ particle and streaming speed $250-750 \mathrm{~km} / \mathrm{s}$ (Meyer-Vernet 2007). The community of space sciences is therefore seeking to understand this significant flow of matter and energy, especially, its interaction with the Earth as well as other planets and their moons (e.g. ElLabany et al. 2006; Moslem 2006; Xie et al. 2013; Popel et al. 2013; Popel et al. 2015; Popel \& Morozova 2017; Lakhina \& Singh 2015; Sreeraj et al. 2018; Moslem et al. 2018, Moslem et al. 2019).

There are planets, like Earth and Saturn, which are largely protected by their own magnetic fields, which deflect most of the charged particles from the solar wind. Saturn is far away, at about 10 AU from the Sun (Kallenrode 1998). At this distance, the solar wind is rarefied with density $0.01-1 \mathrm{~cm}^{-3}$, but still a significant bulk speed e.g., $300-500 \mathrm{~km} / \mathrm{s}$, and an average temperature $\sim 10^{4} \mathrm{~K}$ (Richardson and Smith 2003; Richardson et al. 2004; Whang et al. 2003). Among the moons which rotate around Saturn, Titan has no intrinsic magnetic field but its orbit is within the Saturn magnetic field shield for most orbital period (Backes et al. 2005). It is considered to be the only moon with a substantial ionosphere in the solar system. Titan has no magnetic field, but it retains remnants of Saturn's magnetic field umbrella on the brief occasions when it passes outside Saturn's magnetosphere and it is directly exposed to the solar wind (Bertucci et al. 2015).

In general, the ionospheres of weakly magnetized planets, such as Venus and Mars, as well as unmagnetized moons, such as Titan, are subject to different mechanisms during their interaction with the solar wind. Titan upper ionosphere has a net loss of plasma particles by a rate of $\sim 10^{24}$ ions/s, which is equivalent to 7 tons/Earth day (Coates et al. 2012). This loss may indeed result from the interaction with the solar wind, but a reasonable understanding of this interaction needs evaluations from theoretical models. Titan is just covered by the parent planet Saturn's magnetic field for the most of its periodical orbiting time; the strong magnetic field encompasses the moons, including Titan. However, for a short time roughly about $5 \%$ of its orbiting time, Titan is outer the umbrella of the Saturn's magnetosheath (Garnier et al. 2009), and an important amount 
of charged particles will escape from ionosphere. Our present study is relevant for such transitory times when the magnetization of Titan's ionosphere is rather week, or even for the ion escapes along the magnetic field lines. Future work will investigate the periodical orbiting time inside the Saturn's magnetic field, when ion escapes may be triggered by different mechanisms. Hence, Coates et al (2015) examined the neutral particles dominated regions of the Saturn magnetosphere and locations near several of Saturn's moons. It was found that sunlight ionizes neutrals producing photo-electrons with characteristic energy spectra, and these photo-electrons may be used as tracers of magnetic field morphology. Moreover, the same photo-electrons, relatively energetic, move easily along the magnetic field and enhance plasma escape by setting up a charge separation and implicitly, an ambipolar electric field that acts to extract ions from the ionosphere.

Observations pointed out that Titan ionosphere consists of many ion species in the ionosphere, depending on the altitude (Vuitton et al. 2007; Vuitton et al. 2009). Actually, observations indicated that significant densities of $\mathrm{HCNH}^{+}, \mathbf{C}_{2} \mathbf{H}_{5}^{+}$, and $\mathrm{CH}_{5}^{+}$ have been created deep inside Titan's ionosphere and then transported to high altitude. At high altitude, i.e. $1600-1700 \mathrm{~km}$, there are three dominant species of ions, $\mathrm{HCNH}^{+}, \mathrm{C}_{2} \mathrm{H}_{5}^{+}$, and $\mathrm{CH}_{5}^{+}$with densities $1-7 \mathrm{~cm}^{-3}, 1-10 \mathrm{~cm}^{-3}$, and $1-8 \mathrm{~cm}^{-3}$, respectively (Cravens et al. 2006; Westlake et al. 2012). Salem et al. (2017) have studied the ionized particles escaping from Titan ionosphere at lower altitude, i.e. $\sim 1300 \mathrm{~km}$, where dominant are $\mathrm{HCNH}^{+}$and $\mathrm{C}_{2} \mathrm{H}_{5}^{+}$, and the effect of the solar wind can be neglected. A series of simulations of ion loss from Titan have also been performed, see e.g. Ma et al. 2004; Ma et al. 2006; Tucker \& Johnson 2009; Cui et al. 2010; Lipatov et al. 2014; Richard et al. 2015). Using MHD or hybrid kinetic models provided us with a possible dynamical behavior of the ion escape from Titan ionosphere. It may not be in the interest of understanding of these effects if we take all into account at the same, such as the solar wind effect, different ion masses that were generated inside the ionosphere, etc. The present work may be taken as a complement of the previous works, which may have also considered different ion species along with the solar wind effect. In the present study we consider higher altitudes in the ionosphere of Titan, with electrons and three dominant species of ions, $\mathrm{HCNH}^{+}$, 
$\mathrm{C}_{2} \mathrm{H}_{5}^{+}$, and $\mathrm{CH}_{5}^{+}$which interact with the solar wind electrons and protons. If present, other species of ions have very low densities and can be ignored from a first-order approach. Here we adopt a fluid model mainly motivated by our interest for the large scale dynamics that can explain the expansion of the ionized ionosphere. A fluid approximation cannot be easily justified for the hot and dilute plasmas along Titan's orbit, whether in the outer magnetosphere, magnetosheath or solar wind. These plasmas are not collisional and may exhibit nonthermal features, like kinetic anisotropies and suprathermal populations (Pierrard \& Lazar 2010; Lazar 2012), triggered by the interactions between particles and wave fluctuations. However, the fluid-like dynamics of these plasmas must be triggered by the low-energy (core) populations with a Maxwellian distribution and more than $90 \%$ relative density.

The aim of this work is twofold: (i) to introduce a possible mechanism capable to describe the material loss from Titan upper ionosphere under a direct influence of the solar wind, and (ii) study the prospective plasma parameters which control both the plasma expansion and the mass loss in upper Titan ionosphere.

\section{MODEL EQUATIONS}

Inspired from the observations, our theoretical model for the upper ionosphere of Titan consists of three types of positive ions, namely $\mathrm{HCNH}^{+}, \mathrm{C}_{2} \mathrm{H}_{5}^{+}$, and $\mathrm{CH}_{5}^{+}$(we refer to them with subscripts $j=1,2$, and 3, respectively), and Maxwellian electrons (subscript $k=e$ ), interacting with the solar wind protons $(j=s p)$ and electrons $(k=s e)$. Dynamics of this plasma model can be described by the following fluid equations

$$
\begin{gathered}
\frac{\partial n_{j}}{\partial t}+\frac{\partial}{\partial x} n_{j} u_{j}=0 \\
m_{j} n_{j}\left(\frac{\partial}{\partial t}+u_{j} \frac{\partial}{\partial x}\right) u_{j}+Z_{j} e n_{j} \frac{\partial \varphi}{\partial x}+\frac{\partial P_{j}}{\partial x}=0 \\
e n_{k} \frac{\partial \varphi}{\partial x}-\frac{\partial P_{k}}{\partial x}=0 .
\end{gathered}
$$

We close the system of equations using the following plasma neutrality condition

$$
n_{1}+n_{2}+n_{3}+n_{s p}=n_{e}+n_{s e}
$$

Here, $n_{j}, u_{j}, P_{j}$, and $\varphi$ refer to densities, velocities, thermal pressures, and electric potential, respectively. The system of Eqs. (1)-(4) is closed by the equation of state relating 
$P_{j, k}$ to $n_{j, k}$. We use $P_{j, k} \equiv k_{B} T_{j, k} n_{j, k}^{\Gamma}$, where $\Gamma=(2+N) / N$, and $N$ is the number of degrees of freedom, $k_{B}$ is Boltzmann constant and $T_{j, k}$ is the temperature of different species of particles of mass $m_{j}$ and charge number $Z_{j}$. In one-dimensional adiabatic ion pressures $N=1$ and then $\Gamma=3$ but for isothermal compression electrons $\Gamma=1$.

In order to investigate the plasma expansion from the upper ionosphere, one has to solve the basic Eqs. (1)-(4) numerically. Partial differentiation is not straightforward in this case, and we use a self-similar transformation to describe the plasma expansion in a frame comoving with velocity of ion-acoustic speed $c_{s}=\left(k_{B} T_{e} / m_{1}\right)^{1 / 2}$. This approach is often used to understand various processes in space and laboratory plasmas, such as the expansion of astrophysical objects, laser-plasma interaction, and creation of surface nano-structures (see e.g. Mora 2003; Djebli 2003; Djebli et al. 2004; Moslem 2012; El-Labany et al. 2014; Bennaceur-Doumaz et al. 2015; Elkamash \& Kourakis 2016; Shahmansouri et al. 2017; Moslem et al. 2017). This approach requires a new variable $\xi=x / c_{s} t$, which is used in Eqs. (1)-(4) to obtain the following normalized ordinary differential equations

$$
\begin{gathered}
\left(V_{1}-\xi\right) \frac{d N_{1}}{d \xi}+N_{1} \frac{d V_{1}}{d \xi}=0 \\
3 \sigma_{1} N_{1} \frac{d N_{1}}{d \xi}+\left(V_{1}-\xi\right) \frac{d V_{1}}{d \xi}+\frac{d \phi}{d \xi}=0 \\
\left(V_{2}-\xi\right) \frac{d N_{2}}{d \xi}+N_{2} \frac{d V_{2}}{d \xi}=0 \\
3 Q_{2} \sigma_{2} N_{2} \frac{d N_{2}}{d \xi}+\left(V_{2}-\xi\right) \frac{d V_{2}}{d \xi}+Q_{2} \frac{d \phi}{d \xi}=0 \\
\left(V_{3}-\xi\right) \frac{d N_{3}}{d \xi}+N_{3} \frac{d V_{3}}{d \xi}=0 \\
3 Q_{3} \sigma_{3} N_{3} \frac{d N_{3}}{d \xi}+\left(V_{3}-\xi\right) \frac{d V_{3}}{d \xi}+Q_{3} \frac{d \phi}{d \xi}=0 \\
\frac{d \phi}{d \xi}-\frac{1}{N_{e}} \frac{d N_{e}}{d \xi}=0 \\
\frac{d \phi}{d \xi}-\frac{\sigma_{s e}}{N_{s e}} \frac{d N_{s e}}{d \xi}=0 \\
\left(\widetilde{V}_{s p}-\xi\right) \frac{d N_{s p}}{d \xi}+N_{s p} \frac{d V_{s p}}{d \xi}=0 \\
3 Q_{s p} \sigma_{s p} N_{s p} \frac{d N_{s p}}{d \xi}+\left(\widetilde{V}_{s p}-\xi\right) \frac{d V_{s p}}{d \xi}+Q_{s p} \frac{d \phi}{d \xi}=0
\end{gathered}
$$




$$
\alpha \frac{d N_{1}}{d \xi}+\beta \frac{d N_{2}}{d \xi}+\gamma \frac{d N_{3}}{d \xi}-\frac{d N_{e}}{d \xi}-\delta \frac{d N_{s e}}{d \xi}+\varepsilon \frac{d N_{s p}}{d \xi}=0 .
$$

In Eqs. (5)-(15) we use $\sigma_{1}=T_{1} / T_{e}, \sigma_{2}=T_{2} / T_{e}, \sigma_{3}=T_{3} / T_{e}, \sigma_{s e}=T_{s e} / T_{e}, \sigma_{s p}=T_{s p} / T_{e}$, $Q_{2}=m_{1} / m_{2}, Q_{3}=m_{1} / m_{3}, Q_{s p}=m_{1} / m_{s p}, \alpha=n_{10} / n_{e 0}, \beta=n_{20} / n_{e 0}, \gamma=n_{30} / n_{e 0}, \delta=$ $n_{s e 0} / n_{e 0}$, and $\varepsilon=n_{s p 0} / n_{e 0} . N_{j}$ is the dimensionless density normalized by the unperturbed density $n_{j 0}, \widetilde{V}_{s p}=V_{s p 0}+V_{s p}$ where $V_{s p 0}$ is the initial streaming speed of the solar wind protons, $V_{s p}$ is the dimensionless perturbed velocity normalized by the ion-acoustic speed $c_{s}$, $\phi$ is the dimensionless potential normalized by $k_{B} T_{e} / e$, and $P_{j, k}$ is normalized by $n_{j, k 0} k_{B} T_{j, k}$. Equations (5)-(15) can be rewritten in a matrix form as follows

$$
\begin{aligned}
& \left(\begin{array}{ccccccccccc}
\left(V_{1}-\xi\right) & 0 & 0 & 0 & 0 & 0 & N_{1} & 0 & 0 & 0 & 0 \\
3 \sigma_{1} N_{1} & 0 & 0 & 0 & 0 & 0 & \left(V_{1}-\xi\right) & 0 & 0 & 0 & 1 \\
0 & \left(V_{2}-\xi\right) & 0 & 0 & 0 & 0 & 0 & N_{2} & 0 & 0 & 0 \\
0 & 3 Q_{2} \sigma_{2} N_{2} & 0 & 0 & 0 & 0 & 0 & \left(V_{2}-\xi\right) & 0 & 0 & Q_{2} \\
0 & 0 & \left(V_{3}-\xi\right) & 0 & 0 & 0 & 0 & 0 & N_{3} & 0 & 0 \\
0 & 0 & 3 Q_{3} \sigma_{3} N_{3} & 0 & 0 & 0 & 0 & 0 & \left(V_{3}-\xi\right) & 0 & Q_{3} \\
0 & 0 & 0 & \left(\frac{-1}{N_{e}}\right) & 0 & 0 & 0 & 0 & 0 & 0 & 1 \\
0 & 0 & 0 & 0 & \left(\frac{-\sigma_{s e}}{N_{s e}}\right) & 0 & 0 & 0 & 0 & 0 & 1 \\
0 & 0 & 0 & 0 & 0 & \left(\widetilde{V}_{s p}-\xi\right) & 0 & 0 & 0 & N_{s p} & 0 \\
0 & 0 & 0 & 0 & 0 & 3 Q_{s p} \sigma_{s p} N_{s p} & 0 & 0 & 0 & \left(\widetilde{V}_{s p}-\xi\right) & Q_{s p} \\
\alpha & \beta & \gamma & -1 & -\delta & \varepsilon & 0 & 0 & 0 & 0 & 0
\end{array}\right) \\
& \left(\begin{array}{c}
N_{1}^{\prime} \\
N_{2}^{\prime} \\
N_{3}^{\prime} \\
N_{e}^{\prime} \\
N_{s e}^{\prime} \\
N_{s p}^{\prime} \\
V_{1}^{\prime} \\
V_{2}^{\prime} \\
V_{3}^{\prime} \\
V_{s p}^{\prime} \\
\phi^{\prime}
\end{array}\right)=0
\end{aligned}
$$

where prime indicates the first derivative in variable $\xi$. To solve the basic equations numerically, we need nontrivial solution which is obtained when the following determinant vanishes

$$
\left|\begin{array}{ccccccccccc}
\left(V_{1}-\xi\right) & 0 & 0 & 0 & 0 & 0 & N_{1} & 0 & 0 & 0 & 0 \\
3 \sigma_{1} N_{1} & 0 & 0 & 0 & 0 & 0 & \left(V_{1}-\xi\right) & 0 & 0 & 0 & 1 \\
0 & \left(V_{2}-\xi\right) & 0 & 0 & 0 & 0 & 0 & N_{2} & 0 & 0 & 0 \\
0 & 3 Q_{2} \sigma_{2} N_{2} & 0 & 0 & 0 & 0 & 0 & \left(V_{2}-\xi\right) & 0 & 0 & Q_{2} \\
0 & 0 & \left(V_{3}-\xi\right) & 0 & 0 & 0 & 0 & 0 & N_{3} & 0 & 0 \\
0 & 0 & 3 Q_{3} \sigma_{3} N_{3} & 0 & 0 & 0 & 0 & 0 & \left(V_{3}-\xi\right) & 0 & Q_{3} \\
0 & 0 & 0 & \left(\frac{-1}{N_{e}}\right) & 0 & 0 & 0 & 0 & 0 & 0 & 1 \\
0 & 0 & 0 & 0 & \left(\frac{-\sigma_{s e}}{N_{s e}}\right) & 0 & 0 & 0 & 0 & 0 & 1 \\
0 & 0 & 0 & 0 & 0 & \left(\widetilde{V}_{s p}-\xi\right) & 0 & 0 & 0 & N_{s p} & 0 \\
0 & 0 & 0 & 0 & 0 & 3 Q_{s p} \sigma_{s p} N_{s p} & 0 & 0 & 0 & \left(\widetilde{V}_{s p}-\xi\right) & Q_{s p} \\
\alpha & \beta & \gamma & -1 & -\delta & \varepsilon & 0 & 0 & 0 & 0 & 0
\end{array}\right|=0 .
$$


We use appropriate numerical codes to solve this determinant and select positive solution for $V_{2}$, that refers to the plasma expanding in $+\xi$, and then Eqs. (5)-(15) can be solved numerically.

\section{RESULTS AND DISCUSSIONS}

The analysis focuses on the profiles of density, velocity, and electric potential, which are described in a frame moving with ion-acoustic speed $c_{s}$. One must also realize that a self-similar numerical solution of the above equations presents a series of shortcomings. It characterizes an asymptotic behavior of an unbounded problem, lacking a characteristic length and a characteristic time (no scaling parameter), and it cannot describe a time evolution of the expansion. However solving a system of 11 equations is not straightforward, such that here we have used a self-similar approach. Notice that the electrons have less inertia and they react first, and faster then the positive ions, which follow the electrons just to maintain the quasi-neutrality. An ambipolar electrostatic field between both species will be created causing a plasma expansion. As mentioned above, numerical solution of Eqs. (5)-(15) along with the determinant (17) are the toolbox necessary to identify the behavior of $N_{1}, V_{1}$, and $\phi$ during the plasma expansion. We have used Mathematica (Wolfram) to solve numerically Eqs. (5)-(15) as an initial value problem. Solutions are obtained under the neutrality condition $\alpha=1-\beta-\gamma+\delta-\varepsilon$ for the electric charge. In plasma parametrization we have inspired from the observational data reported in Whang et al. 2003; Richardson and Smith 2003; Richardson et al. 2004; Waite et al. 2009; Garnier et al. 2009, and in our model, the normalized parameters such as density and temperature are employed. These parameters are use as initial conditions and are also mentioned in the figures captions.

Figure (1) illustrates the effect of two relative densities $\delta=n_{s e 0} / n_{e 0}$ and $\varepsilon=n_{s p 0} / n_{e 0}$ on $N_{1}, V_{1}$, and $\phi$. An increase of $\delta$ and $\varepsilon$ leads to a slightly increase of the ion number density $N_{1}$, with mention that an increasing $\varepsilon$ is more effective in enhancing the expansion domain. Furthermore, these ions moves faster in the expansion range and the electric potential becomes more negative for higher $\delta$ and $\varepsilon$. We know that the electric potential of a charged particle is the potential energy divided by the electric charge of the particle, implying that (1) for a negative potential a positive (negative) charge has a relatively lower 
(higher) potential energy, and (2) for a positive electrostatic potential a positive (negative) charge has a higher (lower) potential energy. This behavior can be elucidated as follows. An enhancement of $\varepsilon=n_{s p 0} / n_{e 0}$ means that the initial density of solar wind protons $n_{s p 0}$ increases, i.e., more streaming protons are pumped into the system. The electrons have not enough electrostatic potential to maintain the balance with the enhancement of protons, and a large number of protons screen the electrons, lowering the ampipolar electrostatic potential between electrons and ions. Consequently, the ions cannot expand more, and $N_{1}$ expansion domain decreases. However, increasing $\delta=n_{s e 0} / n_{e 0}$, means an excess of electrons which enhances the ampipolar electrostatic potential between electrons and ions, which triggers the ions to take place behind the electrons increases the expansion domain. For more energetic protons, with a higher (initial) velocity $V_{s p 0}$, and higher temperature ratios $\sigma_{s p, s e}=T_{s p, s e} / T_{e}$, the expansion domain does not change, so we do not show these figures here. We speculate that this behavior may result due to the high contrast between the number densities of the solar wind and the Titan ionosphere, the latter being simply very dense and therefore insensitive to a very dilute solar wind. In other words, in this case the thermal energy pumped by the solar wind protons and electrons into the Titan ionosphere is not enough to change the ions escaping behavior.

Figure (2) shows the influence of the densities ratios $\beta=n_{20} / n_{e 0}$ and $\gamma=n_{30} / n_{e 0}$ on the plasma particles loss from Titan ionosphere. There is a significant positive correlation between an increase of $\beta$ and the increase of the expansion domain, velocity, and electric potential. However, no significant effect on the expansion is found when the density ratio $\gamma$ increases, thus we do not we do not show these results. Increasing $\beta$ enhances the escaping velocity and the electric potential. This could conceivably lead to an increase of the separation charge that determines more electrons to follow, and drag more ions to escape from the ionosphere. The most striking result emerging from these figures shows that the density ratio of the heavier ions has the leading role to control the ions escaping, i.e. by comparison to $\mathrm{CH}_{5}^{+}$ions, $\mathrm{C}_{2} \mathrm{H}_{5}^{+}$ions have a more decisive factor in the loss from Titan ionosphere.

Figure (3) depicts the effect of temperature ratios $\sigma_{1}=T_{1} / T_{e}, \sigma_{2}=T_{2} / T_{e}, \sigma_{3}=T_{3} / T_{e}$ on the expansion variables $N_{1}, V_{1}$, and $\phi$. We assume that $\sigma_{1}=\sigma_{2}=\sigma_{3}=\sigma$, for simplicity. For higher values of $\sigma$ the depletion rate of normalized density increases and the ions move faster, which leads to an extension of the expansion domain. The normalized electrostatic potential increases for higher values of $\sigma$. It is worth to mention that we choose the values 
of the parameters corresponding to the upper region of Titan ionosphere (Waite et al. 2009; Garnier et al. 2009): $T_{e} \approx 150-11000 \mathrm{~K}$ and $n_{e 0} \approx(3-7) \times 10^{7} \mathrm{~m}^{-3}$. These parameters correspond to an electric field $E=\phi\left(k_{B} T_{e} / e \lambda_{D e}\right) \approx 1-20 \mathrm{mV}$, for $\varphi \approx 0.015$ where $\lambda_{D e}=\left(\varepsilon_{0} k_{B} T_{e} / e^{2} n_{e 0}\right)^{1 / 2}$. Therefore, the electric field sets up a charge separation that acts to extract ions from the ionosphere and consequently enhances plasma escaping. On the other hand, the charge separation sets up an ambipolar electric field, which acts to extract ions from the ionosphere. Thus, the magnitude of the electric field (or the potential) is controlled by the average electron kinetic energy and number density. To conclude, the escaping of ions from Titan upper ionosphere is described, taking into account the effects of solar wind electrons and protons. This study is focused on ionic loss from high altitude ionosphere $\sim 1600-1700 \mathrm{~km}$, where three major types of positive ions are presented, namely $\mathrm{HCNH}^{+}, \mathrm{C}_{2} \mathrm{H}_{5}^{+}$, and $\mathrm{CH}_{5}^{+}$. The self-similar technique is used to solve the hydrodynamic fluid equations numerically, with intention to facilitate the analysis and differentiate between different profiles of number density, velocity, and electric potential of the expanded plasma.

To our knowledge, there are plasma processes, involving or not plasma waves, that can trigger the acceleration of ions and implicitly their escape from the ionosphere of Titan. Noticeable are a series of mechanisms like modulational interaction, the pickup of cometary ions, the pickup of helium and oxygen ions of interstellar and planetary origin, etc. (e.g. Valenzuela et al. 1986; Bingham et al. 1991; Popel et al. 1995). One interesting task of the Active Magnetospheric Particle Tracer Explorers (AMPTE) satellite mission was to release neutral barium atoms in the solar wind and to produce an artificial man-made comet. The barium atoms ionized by photoionization extremely rapidly generate a dense, expanding plasma cloud that may interrupt the solar wind flow creating diamagnetic cavities (Valenzuela et al. 1986). Different waves may also be generated by cross-field proton-barium streaming instabilities and cross-field electron currents. The saturation mechanism for these waves is considered to be the modulational instability, that contributes to the electron heating and acceleration, which are consistent with the observations.

Solar wind particles do not have a leading influence on the ionic loss at lower altitudes, but their effects increase with the altitude. The plasma ionosphere parameters have an essential 
role on the plasma escaping. The created electric field extract ions from the ionosphere and consequently increases the ionic outflow. The picture of Titan expansion may still be incomplete, for instance, in circumstances when Saturn may induce a transient magnetization of the Titan ionosphere. Moreover, the effects of suprathermal populations present in space plasmas are not captured by the fluid approach adopted here. However, the results of our first-order approach should stimulate further investigations to address more effects and more complex models of the interaction with the solar wind.. Our theoretical model can describe the ion escape in the absence of magnetic field or along the magnetic field lines, and the plasma expansion is described in a frame co-moving with velocity of ion-acoustic speed. To include the effect of Titan's induced magnetosphere on the ion escape we have to solve Maxwell's equations together with the basic equation (5)-(15) which is out the scope of the present work and it will be considered in our future work.

\section{Acknowledgments}

W.M.M. thanks the Alexander von Humboldt-Stiftung (Bonn, Germany) for financial support of renewed research visit. W.M.M. also thanks Professor Dr R. Schlickeiser for his hospitality. M.L. acknowledges support from DFG - German Research Foundation (project SCHL 201/35-1) and FWO - Vlaanderen (project GOA2316N. 


\section{REFERENCES}

Backes H., Neubauer F. M., Dougherty M. K., Achilleos N., André N., Arridge C. S., Bertucci C., Jones G. H., Khurana K. K., Russell C. T., Wennmacher A.: Sci. 308, 992 $(2005)$

Bennaceur-Doumaz D., Bara D., Djebli M.: Laser Part. Beams 33, 723 (2015)

Bertucci C., Hamilton D. C., Kurth W. S., Hospodarsky G., Mitchell D., Sergis N., Edberg N. J. T., Dougherty M. K.: Geophys. Res. lett. 42, 193 (2015) and references therein

Bingham R., Shapiro V. D., Tsytovich V. N., de Angelis U., Gilman M., Shevchenko V. I.: Phys. Fluids B: Plasma Phys. 3, 1728 (1991)

Coates, A. J., Wellbrock A., Lewis G. R., Arridge C. S., Crary F. J., Young D. T., Thomsen M. F., Reisenfeld D. B., Sittler Jr. E. C., Johnson R. E., Szego K., Bebesi Z., Jones G. H.: J. Geophys. Res. Space Phys. 117, A05324 (2012)

Cui J., Galand M., Yelle R. V., Wahlund J.E., Agren K., Waite Jr. J. H., Dougherty M. K.: J. Geophys. Res., 115, A06314 (2010)

Cravens T. E., Richard M., Ma Y.J., Bertucci C., Luhmann J. G., Ledvina S., Robertson I. P., Wahlund J.E., Agren K., Cui J., MullerWodarg I., Waite J. H., Dougherty M., Bell J., Ulusen D.: J. Geophys. Res., 115, A08319 (2010)

Cravens, T. E., Robertson, I. P., Waite Jr., J. H., Yelle, R. V., Kasprzak, W. T., Keller, C. N., Ledvina, S. A., Niemann, H. B., Luhmann, J. G., McNutt, R. L., Ip, W.-H., De La Haye, V., Mueller-Wodarg, I., Wahlund, J.-E., Anicich, V. G., Vuitton V.: Geophys. Res. Lett. 33, L07105 (2006)

Djebli M.: Phys. Plasmas 10, 4910 (2003)

Djebli M., Annou R., Zerguini T. H.: Phys. Plasmas 11, 2267 (2004)

Elkamash I. S., Kourakis I.: Phys. Rev. E 94, 053202 (2016)

El-Labany S. K., Moslem W. M., Safy F. M.: Phys. Plasmas 13, 082903 (2006)

El-Labany S. K., El-Razek H. N. A., El-Gamish G. A.: Astrophys. Space Sci. 353, 413 (2014)

Garnier P., Wahlund J-E., Rosenqvist L., Modolo R., Agren K., Sergis N., Canu P., Andre M., Gurnett D. A., Kurth W. S., Krimigis S. M., Coates A., Dougherty M., Waite J. H.: Ann. Geophys., 27, 4257 (2009) 
Kallenrode May-Britt, 1998, Space Physics. Springer-Verlag Berlin Heidelberg, p. 276

Lakhina G. S., Singh S. V.: Sol. Phys. 290, 3033 (2015)

Lazar, M.: Astron. Astrophys. 547, A94 (2012)

Lipatov A. S., Sittler Jr. E. C., Hartle R. E., Cooper J. F., Simpson D. G.: Planet. Space Sci. 93-94, 119 (2014)

Ma Y., Nagy A. F., Cravens T. E., Sokolov I. V., Clark J., Hansen K. C.: Geophys. Res. Lett. 31, L22803 (2004).

Ma, Y., Nagy A. F., Cravens T. E., Sokolov I. V., Hansen K. C., Wahlund J.-E., Crary F. J., Coates A. J., Dougherty M. K.: J. Geophys. Res., 111, A05207 (2006)

Meyer-Vernet N., 2007, Basic of the Solar Wind. Cambridge Univ. Press, Cambridge

Mora P.: Phys. Rev. Lett. 90, 185002 (2003)

Moslem W. M.: Phys. Lett.A 351, 290 (2006)

Moslem W. M.: Astrophys. Space Sci. 342, 351 (2012)

Moslem W. M., Rezk S., Abdelsalam U. M., El-Labany S. K.: Adv. Space Res. 61, 2190 (2018)

Moslem W. M., Tolba R. E. Ali S.: Phys. Scr. 94, 075601 (2019)

Moslem W. M., El-Said A. S., Sabry R., Shalouf A., El-Labany S. K., Bahloul H.: Phys. Lett. A 381, 102 (2017)

Pierrard V., Lazar M.: Sol. Phys. 267, 153 (2010)

Popel S. I., Morfill G. E., Shukla P. K., Thomas H.: J. Plasma Phys. 79, 1071 (2013)

Popel S. I., Morozova T. I.: Plasma Phys. Rep. 43, 566 (2017)

Pope1 S. I., Vladimirov S. V., Tsytovich V. N.: Phys. Reports 259, 327 (1995) and references therein

Popel S. I., Zelenyi L. M., Atamaniuk B.: Phys. Plasmas 22, 123701 (2015)

Salem S., Moslem W. M., Radi A.: Phys. Plasmas 24, 052901 (2017)

Shahmansouri M., Bemooni A., Mamun A. A.: J. Plasma Phys. 83, 607 (2017)

Sreeraj T., Singh S. V., Lakhina G. S.: Phys. Plasmas 25, 052902 (2018)

Tucker O. J., Johnson R. E.: Planet. Space Sci. 57, 1889 (2009)

Richard M. S., Cravens T. E., Wylie C., Webb D., Chediak Q., Mandt K., Waite Jr. J. H., Rymer A., Bertucci C., Wellbrock A., Windsor A., Coates A. J.: J. Geophys. Res. Space Phys. 120, 1281 (2015)

Richardson J.D., Smith C.W.: Geophys. Res. Lett. 30, 1206 (2003) 
Richardson, J.D., Wang, C., Burlaga, L.F.: Adv. Space Res. 34, 150 (2004)

Valenzuela A., Haerendel G., Wppl H., Melzner F., Ness H., Reiger E., Stocker .I, Batter 0., Hofner H., Loidl J.: Nature 320, 700 (1986)

Vuitton V., Lavvas P., Yelle R. V., Galand M., Wellbrock A., Lewis G. R., Coates A. J., Wahlund J. E.: Planet. Space Sci. 57, 1558 (2009)

Vuitton V., Yelle R. V., McEwan M.J.: Icarus 191, 722 (2007)

Waite Jr. J. H., Young D. T., Westlake J., Lunine J. I., McKay C. P., Lewis W. S., 2009, in Titan from Cassini-Huygens, Brown R. H., Jean-Pierre Lebreton, Waite J. H., by Springer, Dordrecht Heidelberg, p. 208.

Westlake, J. H., Paranicas, C. P., Cravens, T. E., Luhmann, J. G., Mandt, K. E., Smith, H. T., Mitchell, D. G., Rymer, A. M., Perry, M. E., Waite Jr., J. H., Wahlund, J.-E.: Geophys. Res. Lett. 39, L19104 (2012)

Whang Y. C., Burlaga L. F., Wang Y.-M., Sheeley Jr. N. R.: Astrophys. J. 589, 635 (2003)

Xie L., Li L., Zhang Y., De Zeeuw D. L.: Sci. China Earth Sci. 56, 330 (2013) 


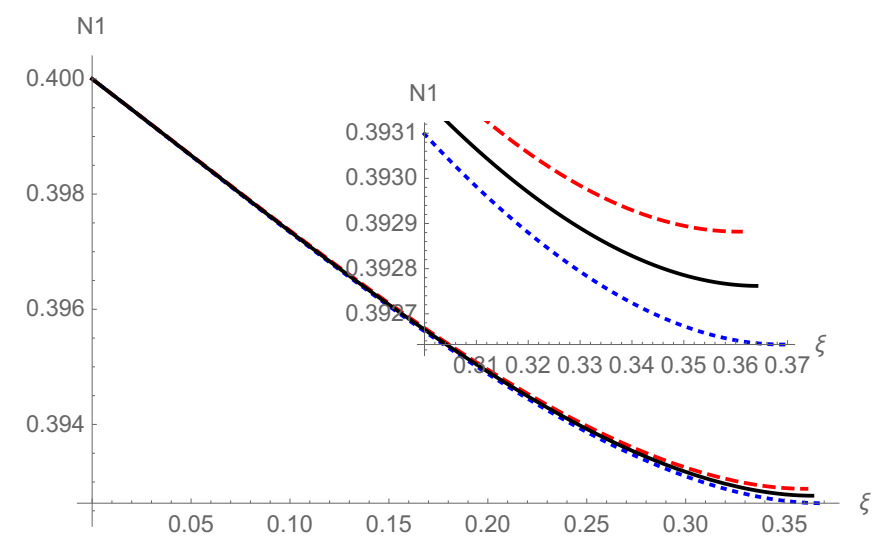

(a)

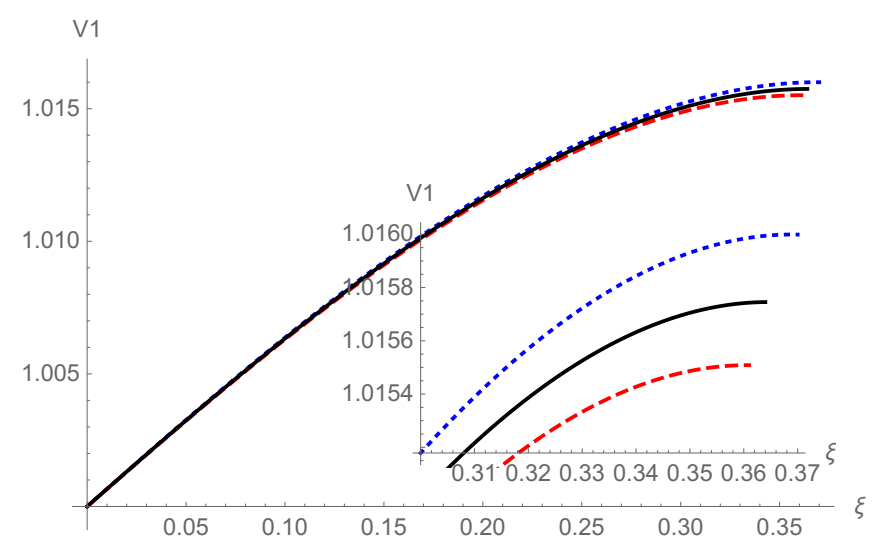

(b)

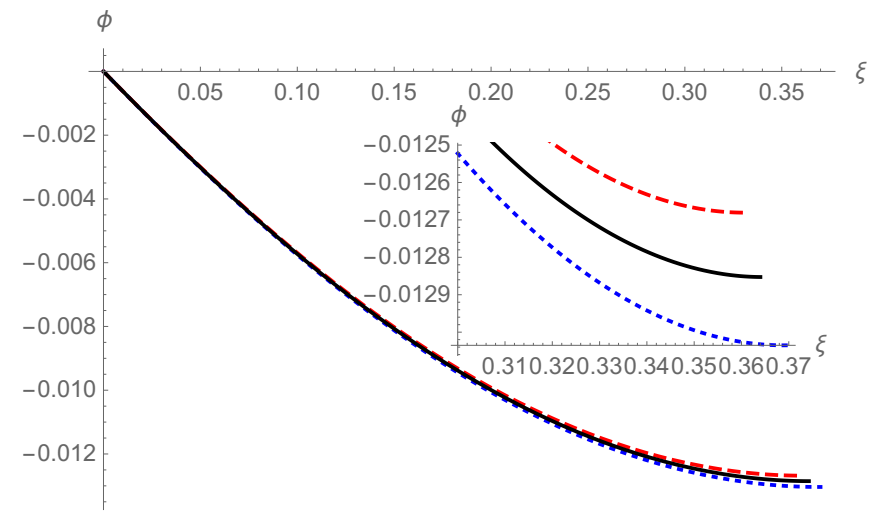

(c)

Figure 1

FIG. 1: (a) Normalized positive ion density $N_{1}$, (b) normalized positive ion velocity $V_{1}$, and (c) normalized electric potential $\phi$ versus the self-similar variable $\xi$, where $\delta=0.001$ and $\varepsilon=0.001$ (solid line), $\delta=0.001$ and $\varepsilon=0.01$ (dashed line), and $\delta=0.01$ and $\varepsilon=0.001$ (dotted line). Here, $Q_{2}=0.9655, Q_{2}=1.647, Q_{s p}=28, \sigma_{1}=\sigma_{2}=\sigma_{3}=0.1, \sigma_{s p}=\sigma_{s e}=4, V_{s p 0}=240, \beta=0.08$, and $\gamma=0.05$. 


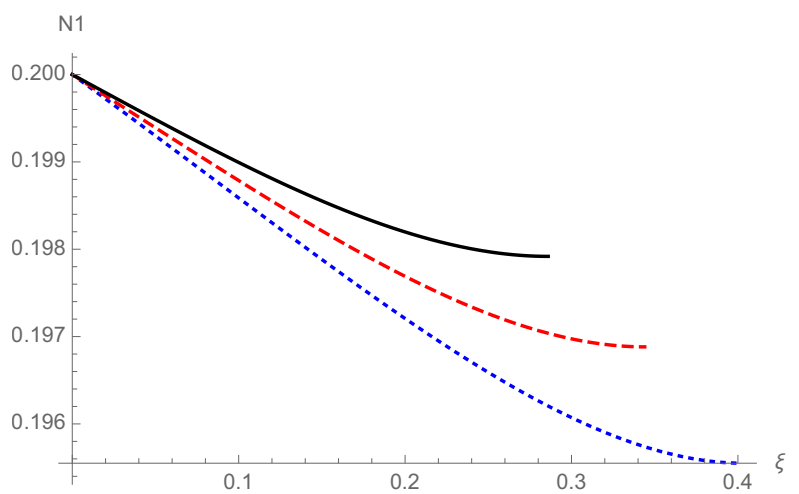

(a)

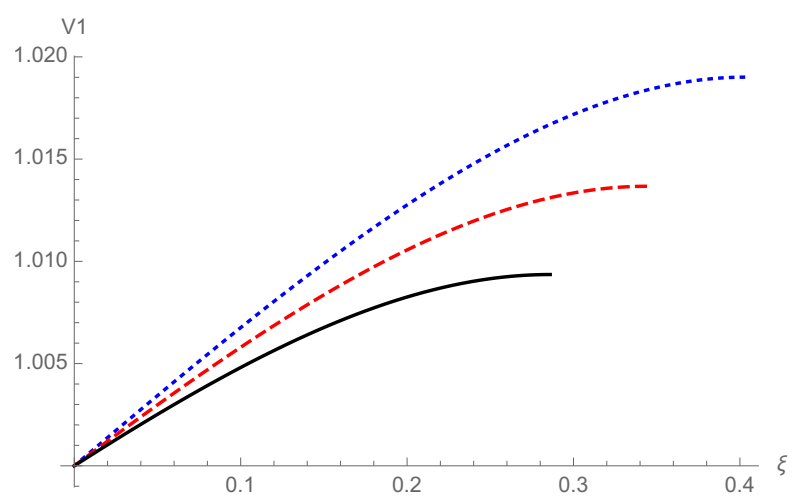

(b)

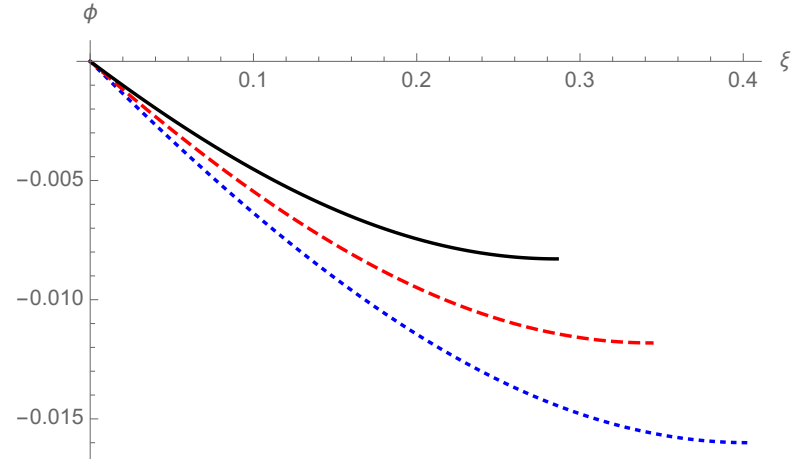

(c)

Figure 2

FIG. 2: (a) Normalized positive ion density $N_{1}$, (b) normalized positive ion velocity $V_{1}$, and (c) normalized electric potential $\phi$ versus the self-similar variable $\xi$, where $\beta=0.08$ (solid line), $\beta=0.09$ (dashed line), and $\beta=0.1$ (dotted line). Here, $Q_{2}=0.9655, Q_{2}=1.647, Q_{s p}=28$, $\sigma_{1}=\sigma_{2}=\sigma_{3}=0.1, \sigma_{s p}=\sigma_{s e}=4, V_{s p 0}=240, \varepsilon=0.01, \delta=0.01$, and $\gamma=0.05$. 


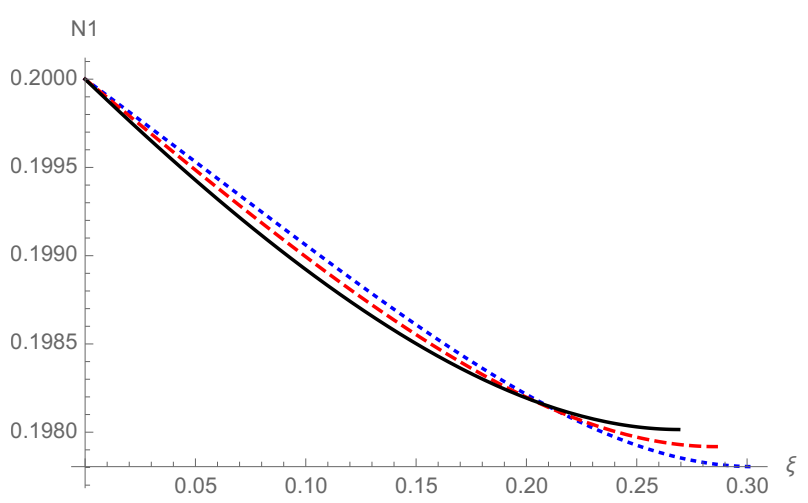

(a)

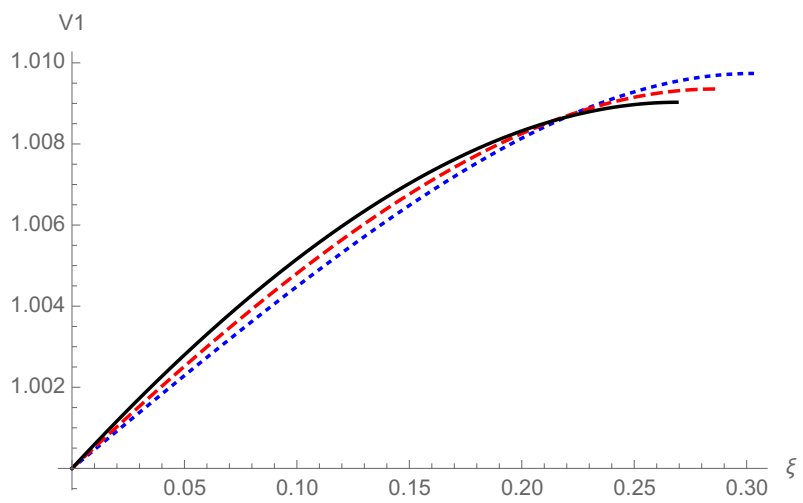

(b)

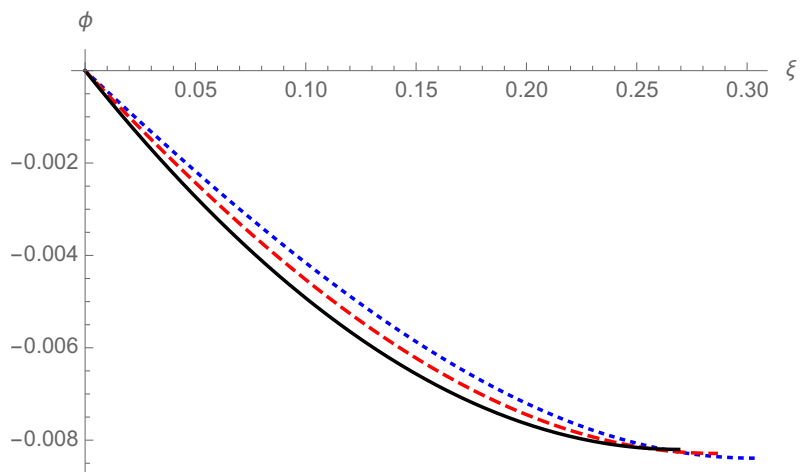

(c)

Figure 3

FIG. 3: (a) Normalized positive ion density $N_{1}$, (b) normalized positive ion velocity $V_{1}$, and (c) normalized electric potential $\phi$ versus the self-similar variable $\xi$, where $\sigma_{1,2,3}=0.01$ (solid line), $\sigma_{1,2,3}=0.1$ (dashed line), and $\sigma_{1,2,3}=0.2$ (dotted line). Here, $Q_{2}=0.9655, Q_{2}=1.647, Q_{s p}=28$, $\sigma_{s p}=\sigma_{s e}=4, V_{s p 0}=240, \varepsilon=0.01, \delta=0.01, \gamma=0.05$, and $\beta=0.08$. 